\title{
Levofloxacin-containing Second-line Anti-Helicobacter pylori Eradication in Taiwanese Real-world Practice
}

\author{
Chih-Ming Liang*, Jen-Wen Cheng*, Chung-Mou Kuo, Kuo-Chin Chang, Keng-Liang Wu, \\ Wei-Chen Tai, King-Wah Chiu, Shue-Shian Chiou, Ming-Tsung Lin, Tsung-Hui Hu, Seng-Kee Chuah
}

Background: Quinolone-containing triple therapy is recommended as an option for non-bismuth containing second-line Helicobacter pylori eradication. Current available Taiwanese reports in the literature used 7-day quinolone-containing triple therapy. As a result, some physicians still prescribe 7-day regimens in real-world practice in Taiwan. This study aimed to further assess the appropriateness of 7-day levofloxacin-containing triple therapy as second-line therapy.

Methods: $\quad$ We enrolled 61 patients who failed $H$. pylori eradication using the standard triple therapy for 7 days and were prescribed levofloxacin-containing second-line triple therapy (levofloxacin $500 \mathrm{mg}$ once daily, amoxicillin $1 \mathrm{~g}$ twice daily, and esomeprazole $40 \mathrm{mg}$ twice daily). Routine follow-up with either endoscopy or urea breath test was done 8 weeks later to assess treatment response.

Results: The eradication rates were $78.7 \%$ in the intention-to-treat analysis and $81 \%$ in the per-protocol analysis. The incidence of adverse events was $6.6 \%$. Drug compliance was 95.1\%. Antibiotic resistance showed the following results:

\begin{abstract}
At a Glance Commentary
Scientific background of the subject

Current available Taiwanese reports in the literature used 7-day quinolone-containing triple therapy as an option for non-bismuth containing second-line $\mathrm{He}$ licobacter pylori eradication. As a result, some physicians still prescribe 7-day regimens in real-world practice in Taiwan.
\end{abstract}

\section{What this study adds to the field}

This study provides an important message that the 7-day levofloxacin-containing triple therapy cannot attain an acceptable per-protocol report card as the second-line treatment for anti-H. pylori eradication in Taiwan and should be modified by either extending the duration to 10-14 days or seeking other regimens. Amoxicillin (0\%), levofloxacin (23.5\%), clarythromycin (35.3\%), metronidazole (17.6\%), and tetracycline $(0 \%)$.

Conclusion: The 7-day levofloxacin-containing triple therapy provides an unacceptable per-protocol report card as the second-line treatment for anti-H. pylori eradication in Taiwan and should be modified by either extending the duration to 10-14 days or seeking other regimens.

(Biomed J 2014;37:326-330)

\section{Key words: 7-day levofloxacin-containing triple therapy, fluoroquinolone, second-line anti-Helicobacter} pylori therapy

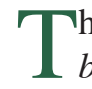
The most widely used second-line therapy for Helicobacter pylori eradication is quadruple therapy, consisting of a proton pump inhibitor (PPI), a bismuth salt, metronidazole, and tetracycline, as recommended by the Maastricht 3-2005 and second Asian Pacific Consensus Report. ${ }^{[1,2]}$ However, bismuth salts are not available worldwide anymore. Alternative second-line therapies with a good treatment report card are still in demand. Antibiotic resistance is one of the important factors in patients' non-responsiveness to initial treatment. ${ }^{[3]}$ Usually, it is not suggested to repeat the same regimens when considering a second-line treatment for H. pylori. ${ }^{[4]}$

\footnotetext{
*Equal contributors.

From the Division of Hepatogastroenterology, Department of Internal Medicine, Kaohsiung Chang Gung Memorial Hospital and Chang Gung University College of Medicine, Kaohsiung, Taiwan

Received: Mar. 22, 2013; Accepted: Oct. 18, 2013

Correspondence to: Dr. Wei-Chen Tai, Division of Hepatogastroenterology, Kaohsiung Chang Gung Memorial Hospital. 123, Ta-Pei Road, Niaosung Dist., Kaohsiung, 833 Taiwan. Tel: 886-7-7317123 ext. 8301; Fax: 886-7-7322402; E-mail: luketai1019@ gmail.com
}

DOI: $10.4103 / 2319-4170.125650$ 
Fluoroquinolone-containing second-line therapy has emerged to be an encouraging strategy for eradication failures owing to the remarkable in vitro activity against H. pylori. ${ }^{[5-9]}$ Besides, levofloxacin-containing triple therapy has the advantage of being good orally absorptive and well tolerated antimicrobial drugs. Moreover, the synergistic effect of quinolone and PPIs on the strains of $H$. pylori enhanced this effect. ${ }^{[6,10]}$ Unfortunately, the existing Taiwanese reports on fluoroquinolone-containing second-line therapy have mentioned unacceptable eradication rates of less than $80 \%{ }^{[11-13]}$ The main drawback in these reports could be the short treatment duration with only a 7-day course.$^{[11-15]}$ Many physicians, especially local medical doctors, still prescribe 7 -day regimens. We, therefore, conducted this retrospective study to determine the appropriateness of 7-day levofloxacin/amoxicillin/PPI regimen as second-line therapy.

\section{METHODS}

\section{Patients and study design}

Sixty-one consecutive $H$. pylori-infected outpatients of at least 18 years of age with endoscopically proven peptic ulcer diseases or gastritis and who failed first-line eradication therapies with standard triple regimens (PPI twice daily, $500 \mathrm{mg}$ of clarithromycin twice daily, and $1 \mathrm{~g}$ of amoxicillin twice daily) were enrolled. Criteria for exclusion included (a) ingestion of antibiotics, bismuth, or PPIs within 4 weeks before; (b) use of nonsteroidal anti-inflammatory drugs within 4 weeks before; (c) patients with allergic history to the medications used; (d) patients with previous gastric surgery; (e) coexistence of serious concomitant illness (e.g., decompensated liver cirrhosis, uremia); (f) pregnant women; and (g) incomplete chart recordings.

\section{Treatment allocation}

These $H$. pylori-infected outpatients who failed first-line eradication therapies were treated with levofloxacin-containing triple therapy (40 mg esomeprazole twice daily, $1 \mathrm{~g}$ of amoxicillin twice daily, and $500 \mathrm{mg}$ of levofloxacin once daily) for 7 days. In all these patients who received anti- $H$. pylori eradication treatment, the patients were asked to return in the $2^{\text {nd }}$ week to assess drug compliance and adverse events, as per our policy. Patients with peptic ulcers in initial endoscopy received an additional 3 weeks of esomeprazole $40 \mathrm{mg}$ orally once daily, while patients with gastritis only took 3 weeks of antacid following eradication therapy. To assess eradication efficacy, repeated endoscopy with rapid urease test and histological examination was performed at 8 weeks after the completion of anti- $H$. pylori therapy. For patients who refused to undergo follow-up endoscopy, urea breath test was used to confirm the $H$. pylori status. The absence of $H$. pylori after a previous eradication therapy was defined as (1) negative results of both rapid urease test and histology or (2) a negative result of urea breath test at 8 weeks. A double-check of urea breath test at 16 weeks was performed for all the participants whose infection were eradicated to avoid possible bias. The technicians who performed the $H$. pylori tests (rapid urease test and urea breath test) or filled in the questionnaires, as well as the pathologists were blinded to the eradication regimens that the patients received. This study was approved by both the Institutional Review Board and the Ethics Committee of Chang Gung Memorial Hospital (IRB101-3169B).

\section{Follow-up for adverse events and compliance}

Since only those patients who were registered as receiving anti- $H$. pylori treatments were enrolled, a complete medical history and demographic data of each patient was available, including age, sex, and medical history, history of smoking, alcohol, and coffee or tea consumption. Smoking was defined as using one pack or more of cigarettes per week. Adverse events such as abdominal pain, diarrhea, constipation, dizziness, taste perversion, headache, anorexia, nausea, vomiting, and skin rash that interfered with patient's quality of life were recorded. Poor compliance was defined as taking less than $80 \%$ of the total medication. ${ }^{[16]}$

\section{Outcomes}

The primary endpoint of our study was successful eradication of $H$. pylori. Additional analyses were conducted on the adverse events during therapies.

\section{Diagnosis of $\boldsymbol{H}$. pylori infection}

\section{Rapid urease test}

One gastric antrum specimen and one corpus biopsy specimen each were collected during endoscopy for testing by using a commercial rapid urease test (Pronto Dry; Medical Instrument Corp., Solothurn, Switzerland). ${ }^{[17]}$ The results of the rapid urease test were interpreted as positive if the color of the gel turned pink or red $1 \mathrm{~h}$ after examination at room temperature.

\section{Urea breath test}

The urea breath test was performed at 8 weeks in accordance with our previous studies. ${ }^{[18]}$ The cut-off value was set at $4.8 \%$ of $\delta 13 \mathrm{CO} 2$. Staffs who performed the tests were blinded to the $H$. pylori status.

\section{Culture and antimicrobial resistance}

Two antral gastric and corpus biopsy specimens each were obtained for isolation of $H$. pylori. $H$. pylori culture was obtained by rubbing the specimens on the surface of 
a Campy-BAP agar plate [Brucella agar (Difco, Sparks, MD, USA) + IsoVitalex (Gibco, Grand Island, NY, USA) $+10 \%$ whole sheep blood]. Then, they were incubated at $37 \mathrm{C}$ under microaerobic conditions $\left(5 \% \mathrm{O}_{2}, 10 \% \mathrm{CO}_{2}\right.$, and $85 \% \mathrm{~N}_{2}$ ) for $4-5$ days. The results were considered positive if one or more colonies of gram-negative bacilli with positive oxidase, catalase, and urease tests were found. The H. pylori strains were tested for tetracycline, metronidazole, and clarithromycin susceptibility using the E-test (AB Biodisk, Solna, Sweden). H. pylori strains with a minimal inhibitory concentration (MIC) values $>4$ $\mu \mathrm{g} / \mathrm{ml}, 8 \mu \mathrm{g} / \mathrm{ml}$, and $>1 \mu \mathrm{g} / \mathrm{ml}$ were considered to be resistant to tetracycline, metronidazole, and clarithromycin, respectively.

\section{Statistical analysis}

Chi-square test with or without Yates correction for continuity and Fisher's exact test were used when appropriate to compare the treatment outcome and host factors using SPSS program (version 10.1, Chicago, IL, USA). A $P$ value less than 0.05 was considered statistically significant.

\section{RESULTS}

\section{Characteristics of the study groups}

A total of $61 \mathrm{H}$. pylori-infected patients who failed first-line therapy were enrolled in our study. The subjects were all included in the intention-to-treat (ITT) analysis for $H$. pylori eradication. The clinical characteristics of patients at entry are summarized in Table 1 . Among the 61 subjects, three patients discontinued medication because of poor compliance to adverse events, and eventually, 58 patients were excluded from per-protocol (PP) analysis for $H$. pylori eradication and they finished all the medications. The eradication rates were $78.7 \%$ in the ITT analysis and $81 \%$ in the PP analysis [Table 2].

\section{Adverse events, complications, and antibiotic resistance}

Interviews regarding the adverse events were carried out in all patients. The incidence of adverse events was $6.6 \%$ and the drug compliance was $95.1 \%$. Dizziness and headache were the most common adverse events [Table 3].

H. pylori strains were isolated from 17 (73.9\%) of the 23 enrolled patients who had undergone endoscopy and bacterial culture in order to check $H$. pylori status on enrollment. Antibiotic resistance results were amoxicillin $(0 \%)$, levofloxacin $(23.5 \%)$, clarythromycin $(35.3 \%)$, metronidazole $(17.6 \%)$, and tetracycline $(0 \%)$.

Univariate analysis showed that none of the clinical factors influenced the efficacy of $H$. pylori eradication. Only $33 \%(1 / 3)$ of patients with poor compliance showed eradi-
Table 1: Demographic data and endoscopic appearance of the patients $(N=61)$

\begin{tabular}{lc}
\hline Characteristics & $\begin{array}{c}\text { 7-day treatment } \\
\text { courses }\end{array}$ \\
\hline Age (years) (mean \pm SD) & $58.6 \pm 14.3$ \\
Gender (male/female) & $24 / 37$ \\
Smoking & $5(8.2 \%)$ \\
Alcohol consumption & $3(4.9 \%)$ \\
Previous history of peptic ulcer & $44(72.1 \%)$ \\
Endoscopic findings & \\
Gastric ulcer & $17(27.9 \%)$ \\
Duodenal ulcer & $16(26.2 \%)$ \\
Gastric and duodenal ulcer & $11(18.0 \%)$ \\
Unspecified (including peptic & $17(27.9 \%)$ \\
ulcer) & \\
Antibiotic sensitivity & \\
(susceptible/resistant) & \\
Amoxicillin & \\
Levofloxacin & $17 / 0(0 \%)$ \\
Clarythromycin & $13 / 4(23.5 \%)$ \\
Metronidazole & $11 / 6(35.3 \%)$ \\
Tetracycline & $14 / 3(17.6 \%)$ \\
\hline
\end{tabular}

Table 2: The major outcomes of $H$. pylori eradication among the patients

\begin{tabular}{lc}
\hline Eradication rate $(N=61)$ & \\
Intention-to-treat & $78.7 \%(48 / 61)$ \\
Per-protocol & $81.0 \%(47 / 58)$ \\
Adverse events & $6.6 \%(4 / 61)$ \\
Compliance & $95.1 \%(58 / 61)$ \\
\hline
\end{tabular}

Table 3: Adverse events among the patients $(N=61)$

\begin{tabular}{lc}
\hline Adverse events & Case number and percentage $(n / \%)$ \\
\hline Abdominal pain & $1(1.64)$ \\
Diarrhea & $0(0)$ \\
Constipation & $0(0)$ \\
Dizziness/headache & $3(4.91)$ \\
Taste pervasion & $0(0)$ \\
Nausea/vomiting & $1(1.64)$ \\
Skin rash & $0(0)$ \\
\hline
\end{tabular}

cation, while $81 \%$ (47/58) with good compliance showed successful eradication [Table 4].

\section{DISCUSSION}

The American College of Gastroenterology Guidelines on the Management of $H$. pylori Infection ${ }^{[19]}$ recommend $^{-1}$ that rescue therapy should consist of a PPI, quinolone, and amoxicillin as one of the options for second-line $H$. pylori eradication which has not been previously used. There are a handful of studies in the literature on levofloxacin in second-line treatment, comparing a levofloxacin-containing regimen with the guideline-recommended second-line quadruple therapy..$^{[-9,20,21]}$ Eradication rates of a levofloxa- 
Table 4: Univariate analysis of the clinical factors influencing the efficacy of $H$. pylori eradication

\begin{tabular}{|c|c|c|c|}
\hline Principal parameter & Case no. & Eradication rate $(\%)$ & $P$ value \\
\hline \multicolumn{4}{|l|}{$\overline{\text { Age }}$} \\
\hline$<60$ years & 33 & $84.8(28)$ & 0.202 \\
\hline 60 years & 28 & $71.4(20)$ & \\
\hline \multicolumn{4}{|l|}{ Sex } \\
\hline Female & 37 & $78.4(29)$ & 0.941 \\
\hline Male & 24 & $79.2(19)$ & \\
\hline \multicolumn{4}{|l|}{ Smoking } \\
\hline$(-)$ & 56 & $78.6(44)$ & 1.000 \\
\hline$(+)$ & 5 & $80.0(4)$ & \\
\hline \multicolumn{4}{|l|}{ Alcohol consumption } \\
\hline$(-)$ & 58 & $79.3(46)$ & 0.519 \\
\hline$(+)$ & 3 & $66.7(2)$ & \\
\hline \multicolumn{4}{|c|}{ Previous history of peptic ulcer } \\
\hline$(-)$ & 17 & $64.7(11)$ & 0.160 \\
\hline$(+)$ & 44 & $84.1(37)$ & \\
\hline \multicolumn{4}{|l|}{ Compliance } \\
\hline Good & 58 & $81.0(47)$ & 0.112 \\
\hline Poor & 3 & $33.3(1)$ & \\
\hline \multicolumn{4}{|c|}{ Amoxicillin resistance } \\
\hline Susceptible & 17 & $64.7(11)$ & - \\
\hline Resistant & - & - & \\
\hline \multicolumn{4}{|c|}{ Levofloxacin resistance } \\
\hline Susceptible & 13 & $69.2(9)$ & 0.584 \\
\hline Resistant & 4 & $50.0(2)$ & \\
\hline
\end{tabular}

cin-containing regimen with treatment duration of 10 days were higher than those of a 7-day treatment course. ${ }^{[22-24]}$ However, currently available Taiwanese reports in the literature used 7-day quinolone-containing triple therapy. As a result, some physicians still prescribe 7-day regimens in actual clinical practice in Taiwan. The results of current study further emphasized the low ITT and PP results with 7-day quinolone-containing triple therapy.

Levofloxacin is a levorotatory isomer of ofloxacin with known activity against many gram-negative and gram-positive bacteria. The mode of action of levofloxacin is based on the inhibition of bacterial DNA topoisomerase II. ${ }^{[25]}$ The advantages of levofloxacin-containing triple therapy are that there is an in vivo synergistic effect of quinolone antimicrobial agents and PPIs on the strains of $\mathrm{H}$. pylori ${ }^{[10]}$ and is simple and well tolerated with a high compliance (95.1\%) as shown in the present study. The relatively low incidence of adverse events of levofloxacin-containing triple therapy was the key factor to the good compliance.

Poor compliance could be caused by the side effects of medicines and poor understanding of the relations between bad compliance and the risk of developing resistant strains. One of the reasons for the high compliance of $95.1 \%$ observed could be the good patient-health care communication status for these registered patients. By doing so, most of these patients, except three, finished all the medications in the present study. However, there was still $66.7 \%$ of patients with poor compliance who failed to eradicate $H$. pylori in the present study.

Antibiotic resistance is one of the important factors for the success of $H$. pylori eradication. Primary resistance to fluoroquinolones is increasing in many parts of the world. ${ }^{[7]}$ The resistance to levofloxacin in a previous Taiwanese report was $21.2 \% .^{[11]}$ The increasing use of quinolones in different countries could be the reason for the increase in resistance to these antibiotics. In the present study, the resistance of levofloxacin was still $23.5 \%$. It is important to realize this issue and the use of levofloxacin should be confined to "rescue" therapy to evade increasing H. pylori resistance toward such an antibiotic. ${ }^{[7-9]}$ The other important reason for failure in previously mentioned studies could be that the duration of fluoroquinolone-containing therapies was too short. Together, these should explain why the eradication rates of the 7-day levofloxacin-containing triple therapy in Taiwan had dropped below $80 \%$, including the present study, even with an increased dose of $750 \mathrm{mg}$ daily in one study. ${ }^{[15]}$

Cytochrome P450 2C19 (CYP2C19) polymorphism of PPIs could play a role in affecting the eradication of H. pylori. Studies with CYP2C19 slow metabolizers have shown that the combination of a PPI plus amoxicillin (dual therapy) can reliably cure more than $90 \%$ of $\mathrm{H}$. pylori infections, especially in Asian populations. ${ }^{[26-29]}$

Generally, clinicians should prescribe therapeutic regimens that have $90 \%$ or greater (grade B level) and probably $95 \%$ or greater (grade A level) PP eradication rate to meet the existing practice in the field of other common bacterial infectious diseases. ${ }^{[30]}$ It is therefore concluded that 7-day levofloxacin-containing triple therapy provides an unacceptable PP report card as the second-line H. pylori eradication treatment in Taiwan and should be modified by either extending the duration to 10-14 days or seeking other regimens.

\section{REFERENCES}

1. Malfertheiner P, Megraud F, O’Morain C, Bazzoli F, El-Omar E, Graham D, et al. Current concepts in the management of Helicobacter infection. The Maastricht III Consensus Report. Gut 2007;56:772-81.

2. Cammarota G, Martino A, Pirozzi G, Cianci R, Branca G, Nista EC, et al. High efficacy of 1-week doxycycline- and amoxicillin-based quadruple regimen in a culture-guided, third-line treatment approach for Helicobacter pylori infection. Aliment Pharmacol Ther 2004;19:789-95.

3. Megraud F. Helicobacter pylori and antibiotic resistance. Gut 2007;56:1502.

4. Parente F, Cucino C, Bianchi Porro G. Treatment options for patients with Helicobacter pylori infection resistant to one or more eradication attempts. Dig Liver Dis 2003;35:523-8. 
5. Zullo A, Hassan C, Lorenzetti R, Morini S. Helicobacter pylori eradication: Do we have another ace up our sleeve? Dig Liver Dis 2001;33:805-6.

6. Croom KF, Goh KL. Levofloxacin: A review of its use in the treatment of bacterial infections in the United States. Drugs 2003;63:2769-802.

7. Wong WM, Gu Q, Chu KM, Yee YK, Fung FM, Tong TS, et al. Lansoprazole, levofloxacin and amoxicillin triple therapy vs. quadruple therapy as second-line treatment of resistant Helicobacter pylori infection. Aliment Pharmacol Ther 2006;23:421-7.

8. Saad RJ, Schoenfeld P, Kim HM, Chey WD. Levofloxacin-based triple therapy versus bismuth-based quadruple therapy for persistent Helicobacter pylori infection: A meta-analysis. Am J Gastroenterol 2006;101:488-96.

9. Chuah SK, Tsay FW, Hsu PI, Wu DC. A new look at anti-Helicobacter pylori therapy. World J Gastroenterol 2011;17:3971-5.

10. Tanaka M, Isogai E, Isogai H, Hayashi S, Hirose K, Kimura K, et al. Synergic effect of quinolone antibacterial agents and proton pump inhibitors on Helicobacter pylori. J Antimicrob Chemother 2002;49:1039-40.

11. Kuo CH, Hu HM, Kuo FC, Hsu PI, Chen A, Yu FJ, et al. Efficacy of levofloxacin-based rescue therapy for Helicobacter pylori infection after standard triple therapy: A randomized controlled trial. J Antimicrob Chemother 2009;63:1017-24.

12. Cheng HC, Chang WL, Chen WY, Yang HB, Wu JJ, Sheu BS. Levofloxacin-containing triple therapy to eradicate the persistent H. pylori after a failed conventional triple therapy. Helicobacter 2007;12:359-63.

13. Hu TH, Chuah SK, Hsu PI, Wu DC, Tai WC, Chiu YC, et al. Randomized comparison of two nonbismuth-containing rescue therapies for Helicobacter pylori. Am J Med Sci 2011;342:177-81.

14. Chuah SK, Hsu PI, Chang KC, Chiu YC, Wu KL, Chou YP, et al. Randomized comparison of two non-Bismuth-containing second-line rescue therapies for Helicobacter pylori. Helicobacter 2012;17:216-23.

15. Liou JM, Lin JT, Chang CY, Chen MJ, Cheng TY, Lee YC, et al. Levofloxacin-based and clarithromycin-based triple therapies as first-line and second-line treatment for helicobacter pylori infection: A randomized comparative trial with crossover design. Gut 2010;59:572-8.

16. Hsu PI, Lai KH, Tseng HH, Lo GH, Lo CC, Lin CK, et al. Eradication of Helicobacter pylori prevents ulcer development in patients with ulcer-like functional dyspepsia. Aliment Pharmacol Ther 2001;15:195-201.

17. Yakoob J, Abid S, Jafri W, Abbas Z, Islam M, Ahmad Z. Comparison of biopsy-based methods for the detection of Helicobacter pylori infection. Br J Biomed Sci 2006;63:159-62.
18. Peng NJ, Lai KH, Liu RS, Lee SC, Tsay DG, Lo CC, et al. Clinical significance of oral urease in the diagnosis of $H$. pylori infection by 13C-urea breath test. Dig Dis Sci 2001;46:1772-8.

19. Chey WD, Wong BC. American College of Gastroenterology guideline on the management of Helicobacter pylori infection. Am J Gastroenterol 2007;102:1808-25.

20. Bilardi C, Dulbecco P, Zentilin P, Reglioni S, Iiritano E, Parodi A, et al. A 10-day levofloxacin-based therapy in patients with resistant Helicobacter pylori infection: A controlled trial. Clin Gastroenterol Hepatol 2004;2:997-1002.

21. Schrauwen RW, Janssen MJ, de Boer WA. Seven-day PPI-triple therapy with levofloxacin is very effective for Helicobacter pylori eradication. Neth J Med 2009;67:96-101.

22. Gisbert JP, Molina-Infante J, Marin AC, Vinagre G, Barrio J, McNicholl AG. Second-line rescue triple therapy with levofloxacin after failure of non-bismuth quadruple "sequential" or "concomitant" treatment to eradicate $H$. pylori infection. Scand J Gastroenterol $2013 ; 48: 652-6$

23. Miehlke S, Krasz S, Schneider-Brachert W, Kuhlisch E, Berning M, Madisch A, et al. Randomized days of esomeprazole, moxifloxacin, and amoxicillin for trial on 14 versus 7 second-line or rescue treatment of Helicobacter pylori infection. Helicobacter 2011;16:420-6.

24. De Sarro A, De Sarro G. Adverse reactions to fluoroquinolones. An overview on mechanistic aspects. Curr Med Chem 2001;8:371-4

25. Marzio L, Coraggio D, Capodicasa S, Grossi L, Cappello G. Role of the preliminary susceptibility testing for initial and after failed therapy of Helicobacter pylori infection with levofloxacin, amoxicillin, and esomeprazole. Helicobacter 2006;11:237-42.

26. De Francesco V, Giorgio F, Hassan C, Manes G, Vannella L, Panella C, et al. Worldwide $H$. pylori antibiotic resistance: A systematic review. J Gastrointestin Liver Dis 2010;19:409-14.

27. Sheu BS, Kao AW, Cheng HC, Hunag SF, Chen TW, Lu CC, et al. Esomeprazole $40 \mathrm{mg}$ twice daily in triple therapy and the efficacy of Helicobacter pylori eradication related to CYP2C19 metabolism. Aliment Pharmacol Ther 2005;21:283-8.

28. Shimizu T, Ochiai H, Asell F, Shimizu H, Saitoh R, Hama Y, et al. Bioinformatics research on inter-racial difference in drug metabolism. Analysis on frequencies of mutant alleles and poor metabolizers on CYP2D6 and CYP2C19. Drug Metab Pharmacokinet 2003;18:48-70.

29. Madan A, Holland S, Humbert JE, Benitz WE. Racial differences in birth weight of term infants in a northern California population. J Perinatol 2002;22:230-5.

30. Graham DY, Lu H, Yamaoka Y. A report card to grade Helicobacter pylori therapy. Helicobacter 2007;12:275-8. 